Louisiana State University

LSU Digital Commons

$9-1-2010$

\title{
Stabilization of a chemostat model with Haldane growth functions and a delay in the measurements
}

\author{
Frédéric Mazenc \\ CNRS Centre National de la Recherche Scientifique \\ Michael Malisoff \\ Louisiana State University
}

Follow this and additional works at: https://digitalcommons.Isu.edu/mathematics_pubs

\section{Recommended Citation}

Mazenc, F., \& Malisoff, M. (2010). Stabilization of a chemostat model with Haldane growth functions and a delay in the measurements. Automatica, 46 (9), 1428-1436. https://doi.org/10.1016/

j.automatica.2010.06.012

This Article is brought to you for free and open access by the Department of Mathematics at LSU Digital Commons. It has been accepted for inclusion in Faculty Publications by an authorized administrator of LSU Digital Commons. For more information, please contact ir@lsu.edu. 


\title{
Stabilization of a Chemostat Model with Haldane Growth Functions and a Delay in the Measurements
}

\author{
Frédéric Mazenc $^{\mathrm{a}}$ and Michael Malisoff ${ }^{\mathrm{b}}$ \\ ${ }^{a}$ EPI MERE INRIA-INRA, UMR Analyse des Systèmes et Biométrie, INRA 2, place Viala, 34060 Montpellier, France \\ ${ }^{\mathrm{b}}$ Dept. of Mathematics, Louisiana State University, Baton Rouge, LA 70803-4918
}

\begin{abstract}
The stabilization of equilibria in chemostats with measurement delays is a complex and challenging problem, and is of significant ongoing interest in bioengineering and population dynamics. In this paper, we solve an output feedback stabilization problem for chemostat models having two species, one limiting substrate, and either Haldane or Monod growth functions. Our feedback stabilizers depend on a given linear combination of the species concentrations, which are both measured with a constant time delay. The values of the delays are unknown. Instead, one only knows an upper bound on the delays, and we allow the upper bound to be arbitrarily large. The stabilizing feedback depends on the known upper bound for the delays as well. Our work is based on the construction of a Lyapunov-Krasovskii functional.
\end{abstract}

Key words: Delayed systems, systems biology, uniform global asymptotic stability

\section{Introduction}

The chemostat model plays a central role in current research in bioengineering, microbial ecology, and population biology [6],[14],[16],[18],[20]. For two species chemostats with one limiting substrate, and with the yield coefficients taken equal to one without loss of generality, the model has the form [19]

$$
\left\{\begin{array}{c}
\dot{s}=\left(s_{\text {in }}-s\right) D-\sum_{i=1}^{2} \mu_{i}(s) x_{i} \\
\dot{x}_{i}=\left[\mu_{i}(s)-D\right] x_{i}, i=1,2,
\end{array}\right.
$$

where the $x_{i}$ 's are the concentrations of the species, $s$ is the level of the substrate, $D$ is an everywhere positive dilution rate controller (which we specify below), $s_{\text {in }}>0$ is the constant input nutrient concentration, and the $\mu_{i}$ 's are the growth functions. The dynamics (1) models two competing species growing on one substrate in a well stirred biological reactor. Since the function $D$ is the ratio of the volumetric flow rate (with units of volume over

\footnotetext{
ऋ Malisoff was supported by AFOSR Grant FA9550-09-10400 and NSF DMS Grant 0708084. The authors thank Jérôme Harmand and Patrick De Leenheer for illuminating discussions. A summary of this paper is in preparation for submission to the 2010 American Control Conference.

Email addresses: Frederic. Mazenc@supagro.inra.fr (Frédéric Mazenc), malisoff@lsu.edu (Michael Malisoff).
}

time) to the constant reactor volume, it is proportional to the speed of the pump that supplies the reactor with fresh nutrient. In classical cases where $D$ is constant, the well known competitive exclusion principle says that only one species survives in an equilibrium [19]. In this paper, we wish to stabilize a componentwise positive equilibrium for (1) (i.e., persistence of both species), so we must take $D$ to be a nonconstant controller.

There are several commonly used expressions for the growth functions. One important choice is the Haldane function

$$
\mu_{i}(s)=\frac{K_{i} s}{L_{i}+s+g_{i} s^{2}},
$$

where $g_{i}$ is a nonnegative constant, and where $K_{i}$ and $L_{i}$ are positive constants for $i=1,2$. Growth functions of the type (2) for which $g_{i}=0$ are called Monod growth functions [19]. However, it is often useful to allow positive $g_{i}$ 's, to model more realistic cases where an excessive level of the substrate $s$ can impede the growth $[5,11]$.

In our earlier work [16], we designed a controller $D$, depending only on a linear combination $Y(t)=x_{1}(t)+$ $a x_{2}(t)$ of the species levels and with an arbitrary but known constant $a \in(0,1)$, that ensures that (1) is globally asymptotically stable to an appropriate equilibrium $\left(s_{*}, x_{1 *}, x_{2 *}\right) \in(0, \infty)^{3}$. However, [16] only applied to cases where the $\mu_{i}$ 's are Monod, and did not allow any 
measurement delay. See [10] for a thorough stability analysis for one species chemostats with general growth functions but with no delays, and $[8,9]$ for significant results for undelayed chemostats under (possibly unbounded) state feedback. It is important to allow delays, because measurements may take time. See [18], which makes a strong case for delays only in the dilution rate controller $D$ and gives a stability analysis for one species chemostats with delays in $D$. In this paper, we analyze the more complex case of two species, where $D$ now depends on a delayed linear combination $y(t)=x_{1}(t-\tau)+a x_{2}(t-\tau)$ of the $x_{i}$ 's and $\tau \geq 0$ is an unknown delay.

As in [13],[14],[15],[16], we design $D$ to globally asymptotically stabilize a componentwise positive equilibrium triple. However, when there are time delays, the strict Lyapunov function approach in [13],[14],[15],[16] does not apply. See also $[1,2]$ for an alternative feedback linearization method for two species chemostats, and see Remark 4 below for more information on the relationship between this work and our previous results. By contrast, here we present a new Lyapunov-Krasovskii construction. The construction uses the formulas for the $\mu_{i}$ 's and is reminiscent of the Lyapunov function under a constant dilution rate that was used in [7].

Our control design is very different from the one in [3]. The advantage of [3] is that it does not require any specific formula for the $\mu_{i}$ 's. However, [3] uses a PoincaréBendixson approach that does not allow measurement delays and it assumes monotonicity of the $\mu_{i}$ 's. The present paper and [14],[15],[16] all use the analytic expression of the growth functions. The advantage of our new work is that for any given upper bound $\tau_{M}$ on the delay $\tau$, we obtain uniform globally asymptotically stabilizing control laws depending only on the delayed output $y(t)$ and $\tau_{M}$. By allowing feedbacks that only involve a linear combination of the species concentrations, we cover cases where the measurements are done using photometric methods that may not distinguish between the species; see $[4,16]$ for details and more motivation for the important setting where only a linear combination of the $x_{i}$ 's can be measured.

\section{Assumptions, Definitions, and Notation}

Consider the two species chemostat (1) with a constant $s_{\text {in }}>0$, the growth rates (2), and a bounded controller $D$ to be determined that is bounded from below by a positive constant and dependent only on the unique output

$$
y(t)=x_{1}(t-\tau)+a x_{2}(t-\tau) .
$$

Here $a \in(0,1)$ is any given constant and the constant time delay $\tau \geq 0$ is unknown. We always assume:

Assumption 1 There is a constant $s_{*} \in\left(0, s_{\text {in }}\right)$ such that

$$
\mu_{1}\left(s_{*}\right)=\mu_{2}\left(s_{*}\right) .
$$

Therefore, we are free to fix any constants $x_{1 *}>0$ and $x_{2 *}>0$ such that $s_{*}+x_{1 *}+x_{2 *}=s_{\text {in }}$. Set $D_{*}=\mu_{1}\left(s_{*}\right)$. However, the constants are assumed to satisfy:

Assumption 2 The constants

$$
\delta_{i} \stackrel{\text { def }}{=} L_{i}-g_{i} s_{*} s_{\text {in }}, i=1,2
$$

are positive. Also,

$$
\begin{aligned}
\aleph \stackrel{\text { def }}{=} & \delta_{1} D_{*}\left[1+\frac{1}{s_{*}} \sum_{i=1}^{2} \frac{\delta_{i}}{L_{i}+s_{\text {in }}+g_{i} s_{\text {in }}^{2}} x_{i *}\right] \\
& +K_{2} s_{*} x_{2 *} \frac{L_{2} g_{1}-L_{1} g_{2}}{\delta_{2}}
\end{aligned}
$$

is positive, $L_{2} g_{1}-L_{1} g_{2} \leq 0$, and

$$
\mho \stackrel{\text { def }}{=} \frac{s_{*}}{(1-a) D_{*}}\left[-K_{1}+\frac{L_{1}-g_{1} s_{*}^{2}}{L_{2}-g_{2} s_{*}^{2}} K_{2}\right]
$$

is nonzero.

Assumption $3 \min \left\{\mu_{1}\left(s_{\text {in }}\right), \mu_{2}\left(s_{\text {in }}\right)\right\}>D_{*}$.

Therefore, we can also fix a constant $p \in(0,1)$ so that

$$
\mu_{i}\left(s_{\text {in }}\right)>(1+p) D_{*} \text { for } i=1,2 .
$$

Finally, we assume that there is a known bound $\tau_{M}$ on the unknown constant delay $\tau$ :

Assumption 4 There is a known constant $\tau_{M}>0$ so that $0 \leq \tau \leq \tau_{M}$.

Since we wish to stabilize the triple $\left(s_{*}, x_{1 *}, x_{2 *}\right)$ using a delayed feedback, it is also convenient to define

$$
\begin{gathered}
z=s+x_{1}+x_{2}, \quad \tilde{z}=z-s_{\text {in }}, \\
\tilde{s}=s-s_{*}, \tilde{x}_{i}=x_{i}-x_{i *}(i=1,2), \\
\tilde{y}(t)=\tilde{x}_{1}(t-\tau)+a \tilde{x}_{2}(t-\tau), \\
Y(t)=x_{1}(t)+a x_{2}(t), \text { and } \\
\tilde{Y}(t)=\tilde{x}_{1}(t)+a \tilde{x}_{2}(t) .
\end{gathered}
$$

Then $\tilde{z}=s+x_{1}+x_{2}-\left(s_{*}+x_{1 *}+x_{2 *}\right)=\tilde{s}+\tilde{x}_{1}+\tilde{x}_{2}$.

Remark 1 Assumptions 1-3 automatically hold in the Monod case (where $g_{1}=g_{2}=0$ ) if the $\mu_{i}$ 's intersect at a unique positive level $s_{*} \in\left(0, s_{\text {in }}\right)$. In the Monod case, nonvanishing of $\mho$ is equivalent to the condition $K_{1} / L_{1} \neq K_{2} / L_{2}$, which is the case when the growth functions have a unique positive intersection value $s_{*}$. If our conditions $\aleph>0$ and $\mho \neq 0$ hold for a given pair of Monod growth functions $\mu_{i}(s)=K_{i} s /\left(L_{i}+s\right)$ having a unique positive intersection, then these two assumptions also hold for the Haldane growth functions (2) when the constants $g_{i}$ 's are positive but small enough relative to the other parameters. Therefore, our work can be viewed as a robustness analysis with respect to measurement delays and nonmonotone $\mu_{i}$ 's (corresponding to nonzero constants $g_{i}$ ). 
Remark 2 The requirement that $\mho \neq 0$ cannot be removed. If all of the requirements in Assumption 1-4 hold except that $\mho=0$, and if $\tau=0$ and $\mu_{1} \neq \mu_{2}$, then it is impossible to render $\left(s_{*}, x_{1 *}, x_{2 *}\right)$ globally uniformly asymptotically stable, even if we allow a time dependent controller D; see Appendix A.4.

Given an open subset $\mathcal{G}$ of a Euclidean space containing the origin, a function $\alpha:[0, \infty) \times \mathcal{G} \rightarrow[0, \infty)$ is called positive definite (on $\mathcal{G}$ ) provided $\alpha(t, 0)=0$ for all $t \geq 0$ and $\inf _{t \geq 0} \alpha(t, \zeta)>0$ for all $\zeta \in \mathcal{G} \backslash\{0\}$. A modulus with respect to $\mathcal{G}$ is any continuous function $\alpha: \mathcal{G} \rightarrow[0, \infty)$ satisfying: (A) $\lim _{\zeta \rightarrow \zeta^{*}} \alpha(\zeta)=\infty$ for each point $\zeta^{*}$ in the boundary of $\mathcal{G}$ and $(\mathrm{B}) \lim _{|\zeta| \rightarrow \infty} \alpha(\zeta)=\infty$, where $|\cdot|$ is the usual Euclidean norm. Condition (A) holds vacuously if $\mathcal{G}=\mathbb{R}^{n}$, and (B) holds vacuously if $\mathcal{G}$ is bounded. A function $V$ for which $\zeta \mapsto \inf _{t} V(t, \zeta)$ is a modulus with respect to $\mathcal{G}$ is called proper (on $\mathcal{G}$ ). Also, $C(A, B)$ is the set of all continuous functions $\phi: A \rightarrow B$ for any subsets $A$ and $B$ of Euclidean spaces.

\section{Main Result}

\subsection{Statement of Theorem}

Our desire to stabilize $\left(s_{*}, x_{1 *}, x_{2 *}\right) \in(0, \infty)^{3}$ leads to our designing a nonconstant dilution rate controller

$$
D=D_{*}-\operatorname{sign}(\mho) \varepsilon_{1} \sigma\left(\varepsilon_{2}\left(Y(t-\tau)-x_{1 *}-a x_{2 *}\right)\right)
$$

for small enough constants $\varepsilon_{i}>0$, where $\sigma: \mathbb{R} \rightarrow$ $[-1,+1]$ is the standard saturation defined by $\sigma(r)=r$ if $|r| \leq 1$ and $\sigma(r)=\operatorname{sign}(r)$ otherwise. To specify the bounds on the admissible $\varepsilon_{i}$ 's, we use the following constants, whose choices will be explained where they are used in the proof of our theorem:

$$
\begin{aligned}
& \bar{c}_{1} \stackrel{\text { def }}{=} \\
& \frac{\left(L_{1}-g_{1} s_{*}^{2}\right)\left(s_{\text {in }}-s_{*}\right)}{s_{*}}+\frac{1}{1-a} \frac{s_{*}}{D_{*}}\left[-K_{1} a+\frac{L_{1}-g_{1} s_{*}^{2}}{L_{2}-g_{2} s_{*}^{2}} K_{2}\right], \\
& \bar{c}_{2} \stackrel{\text { def }}{=} \\
& 1+180(1+a)^{2} s_{\text {in }}^{2}|\mho|\left(\max \left\{\frac{D_{*}^{2} s_{\text {in }}}{s_{*}^{2}}, \frac{\bar{c}_{1}^{2} s_{\text {in }}}{2 \aleph}\right\}+1\right)^{2}, \\
& \bar{\varepsilon}_{1} \stackrel{\text { def }}{=} \min \left\{0.5 p D_{*}, \frac{\aleph s_{*}}{10\left(L_{1}+g_{1} s_{*}^{2}\right) s_{\text {in }}}\right\}, \bar{\varepsilon}_{2} \stackrel{\text { def }}{=} \frac{10}{11 s_{\text {in }}}, \\
& \bar{\varepsilon}_{3} \stackrel{\text { def }}{=} \min \left\{\frac{\aleph|\mho|}{5\left(\bar{c}_{1}^{2}+1\right) s_{\text {in }}},|\mho|, \frac{1}{10 \bar{c}_{2} \tau_{M}} \min \left\{\frac{\aleph}{\tau_{M}}, \sqrt{|\mho|}\right\}\right\} .
\end{aligned}
$$

Our main result is:

Theorem 1 If (1) satisfies Assumptions 1-4, then for any positive constants $\varepsilon_{1}$ and $\varepsilon_{2}$ such that $\varepsilon_{i} \leq$ $\bar{\varepsilon}_{i}$ for $i=1,2$ and $\varepsilon_{1} \varepsilon_{2} \leq \bar{\varepsilon}_{3}$, the control law (12) globally asymptotically stabilizes the equilibrium point $\left(s_{*}, x_{1 *}, x_{2 *}\right)$ of (1) for all initial functions $\left(\phi_{s}, \phi_{x_{1}}, \phi_{x_{2}}\right) \in C\left(\left[-2 \tau_{M}, 0\right],(0, \infty)^{3}\right)$.

Remark 3 Our proof of Theorem 1 shows that the $\left(\tilde{s}, \tilde{x}_{1}, \tilde{x}_{2}\right)$ dynamics, in closed loop with (12), is uniformly globally asymptotically stable (UGAS) to 0 on its invariant set, which gives persistence of both species; see Appendix A.3 for the form for the UGAS estimate. Theorem 1 goes beyond asserting existence of a stabilizing delayed feedback of the form (12). Instead, it computes bounds on the allowable $\varepsilon_{i}$ 's. When $\tau_{M} \rightarrow \infty$, we get $\bar{\varepsilon}_{3} \rightarrow 0$ (giving slower convergence to the equilibrium), so in the limiting case, we have a constant controller on any bounded invariant set, giving competitive exclusion. This can be interpreted as saying that the closed loop system has a bifurcation when $\varepsilon_{3}$ 's converges to zero that significantly changes the equilibrium behavior.

\subsection{Outline of Proof}

We first find an appropriate nonstrict control-Lyapunov function $V$ for the open loop error dynamics for $\left(\tilde{s}, \tilde{x}_{1}, \tilde{x}_{2}\right)$ obtained by setting $D=D_{*}+v$, viewing $v$ as the control. This is done in Section 4. Then in Section 5.1, we transform $V$ into an appropriate function $V^{\sharp}$ that is a strict Lyapunov function for the error dynamics, when we choose the feedback (12) and when the delays are zero; see [12] for the standard definitions of strict and nonstrict Lyapunov functions. Finally, in Section 5.2, we transform $V^{\sharp}$ into a functional $U_{1}$ that satisfies the usual Lyapunov-Krasovskii functional properties for the closed loop error dynamics under delays, provided $t \geq \tau_{M}$. This leads to the desired UGAS estimate; see Remark 4 for a comparison of this transformation process to earlier Lyapunov transformation processes in the literature.

\section{Main Lemmas}

In all of what follows, all (in)equalities should be understood to hold globally, unless otherwise indicated, and $C_{\text {in }}=C\left(\left[-2 \tau_{M}, 0\right],(0, \infty)^{3}\right)$ is our set of initial functions. Writing (12) as $D=D_{*}+v$, where $D_{*}=\mu_{1}\left(s_{*}\right)=$ $\mu_{2}\left(s_{*}\right)$ as before, gives $|v| \leq 0.5 p D_{*}$ and therefore also $(1+0.5 p) D_{*} \geq D \geq(1-0.5 p) D_{*}$ everywhere, by our choice of the bound $\bar{\varepsilon}_{1}$ in (13). Also,

$$
\dot{\tilde{z}}=-D \tilde{z}
$$

along all trajectories of (1). Hence, the time derivative of $Q(\tilde{z})=\frac{1}{2} \tilde{z}^{2}$ along the trajectories of (1) satisfies

$$
\dot{Q}=-2 D Q(\tilde{z}) \leq-2(1-0.5 p) D_{*} Q(\tilde{z}) .
$$

Since $D_{*}>0$ and $p \in(0,1)$, this gives $\tilde{z} \rightarrow 0$ exponentially. Moreover, forward completeness of (1) in closed loop with (12) follows from the proof of [17, Lemma A.1] and Lipschitzness of the feedback.

From the structure of the dynamics (1) and the positive lower bound on $D$, it is clear that $s(t)$ must converge towards $\left(0, s_{\text {in }}\right]$ when it starts above $s_{\text {in }}$, for all initial functions in $C_{\mathrm{in}}$. The next lemma implies that $s(t)$ must in fact enter $\left(0, s_{\text {in }}\right)$, and uses Assumption 3. 
Lemma 1 For all initial functions in $C_{\mathrm{in}}$, the set

$$
S=\left\{\left(s, x_{1}, x_{2}\right) \in\left(0, s_{\text {in }}\right) \times(0, \infty)^{2}: z \leq 1.1 s_{\text {in }}\right\}
$$

is positively invariant and globally attractive for the system (1) in closed loop with $D$ defined in (12).

See Appendix A.1 for the proof of Lemma 1. From the definition of $D_{*}$ and the relations

$$
\mu_{i}(s)-D_{*}=\frac{K_{i} s}{L_{i}+s+g_{i} s^{2}}-\frac{K_{i} s_{*}}{L_{i}+s_{*}+g_{i} s_{*}^{2}},
$$

one can readily check that:

Lemma 2 For $i=1,2$ and all $s>0$, we have

$$
\mu_{i}(s)-D_{*}=\frac{D_{*}\left[L_{i}-g_{i} s_{*} s\right]}{s_{*}\left(L_{i}+s+g_{i} s^{2}\right)} \tilde{s} .
$$

Moreover, since $\dot{s}=\dot{\tilde{s}}$ and $\mu_{i}\left(s_{*}\right)=D_{*}$ for $i=1,2$,

$$
\begin{gathered}
\dot{\tilde{s}}=v\left(s_{\text {in }}-s\right)+\left(s_{\text {in }}-\tilde{s}-s_{*}\right) D_{*}-\sum_{i=1}^{2} D_{*} x_{i *} \\
-\sum_{i=1}^{2}\left(\mu_{i}(s)-D_{*}\right) x_{i *}-\sum_{i=1}^{2} \mu_{i}(s) \tilde{x}_{i} .
\end{gathered}
$$

Hence, the fact that $s_{\text {in }}=s_{*}+x_{1 *}+x_{2 *}$ and (17) give

$$
\begin{aligned}
\dot{\tilde{s}}= & -D_{*} \tilde{s}-\sum_{i=1}^{2} \frac{D_{*}\left[L_{i}-g_{i} s_{*} s\right]}{s_{*}\left(L_{i}+s+g_{i} s^{2}\right)} \tilde{s} x_{i *} \\
& -\sum_{i=1}^{2} \frac{K_{i} s}{L_{i}+s+g_{i} s^{2}} \tilde{x}_{i}+\left(s_{\text {in }}-s\right) v .
\end{aligned}
$$

We readily conclude from our choices of the $\delta_{i}$ 's in Assumption 2 that:

Lemma 3 The controller (12) guarantees that along all trajectories of (1), we have

$$
\dot{\tilde{s}}=-H(s) \tilde{s}-\sum_{i=1}^{2} \frac{K_{i} s}{L_{i}+s+g_{i} s^{2}} \tilde{x}_{i}+\left(s_{\mathrm{in}}-s\right) v,
$$

where

$$
H(s)=D_{*}+\sum_{i=1}^{2} \frac{D_{*}\left[L_{i}-g_{i} s_{*} s\right]}{s_{*}\left(L_{i}+s+g_{i} s^{2}\right)} x_{i *} .
$$

Moreover,

$$
H(s) \geq D_{*}\left[1+\frac{1}{s_{*}} \sum_{i=1}^{2} \frac{\delta_{i} x_{i *}}{L_{i}+s_{\mathrm{in}}+g_{i} s_{\mathrm{in}}^{2}}\right]
$$

for all $s \in\left[0, s_{\text {in }}\right]$.

Combining Lemmas 1-3 shows that the error dynamics

$$
\left\{\begin{array}{l}
\dot{\tilde{s}}=-H(s) \tilde{s}-\sum_{i=1}^{2} \frac{K_{i} s}{L_{i}+s+g_{i} s^{2}} \tilde{x}_{i}+\left(s_{\mathrm{in}}-s\right) v \\
\dot{\tilde{x}}_{i}=D_{*} \frac{L_{i}-g_{i} s_{*} s}{s_{*}\left(L_{i}+s+g_{i} s^{2}\right)} \tilde{s} x_{i}-v x_{i}, i=1,2
\end{array}\right.
$$

has the globally attractive and positively invariant set $\mathcal{E}=\left\{\left(\tilde{s}, \tilde{x}_{1}, \tilde{x}_{2}\right) \in\left(-s_{*}, s_{\text {in }}-s_{*}\right) \times\left(-x_{1 *}, \infty\right) \times\right.$ $\left.\left(-x_{2 *}, \infty\right): \tilde{z} \leq 0.1 s_{\text {in }}\right\}$ for all initial functions in $C_{\text {in }}$. To simplify the exposition, we set

$$
C_{1}=K_{1} \frac{s_{*}}{D_{*}} \text { and } C_{2}=\frac{s_{*}}{D_{*}} \frac{K_{2}\left(L_{1}-g_{1} s_{*}^{2}\right)}{L_{2}-g_{2} s_{*}^{2}}
$$

and we use the function

$$
\begin{aligned}
& M\left(\tilde{s}, \tilde{x}_{1}, \tilde{x}_{2}\right) \\
& =\tilde{s} \frac{L_{1}-g_{1} s_{*} s}{s}\left(s_{\mathrm{in}}-s\right)-K_{1} \frac{s_{*}}{D_{*}} \tilde{x}_{1}-K_{2} \frac{s_{*}}{D_{*}} \frac{L_{1}-g_{1} s_{*}^{2}}{L_{2}-g_{2} s_{*}^{*}} \tilde{x}_{2} .
\end{aligned}
$$

Our nonstrict control-Lyapunov construction is:

\section{Lemma 4 The function}

$$
\begin{aligned}
V\left(\tilde{s}, \tilde{x}_{1}, \tilde{x}_{2}\right)= & C_{1} \int_{0}^{\tilde{x}_{1}} \frac{\ell}{\ell+x_{1 *}} \mathrm{~d} \ell+C_{2} \int_{0}^{\tilde{x}_{2}} \frac{\ell}{\ell+x_{2 *}} \mathrm{~d} \ell \\
& +\int_{0}^{\tilde{s}} \frac{\left|L_{1}-g_{1} s_{*}\left(s_{*}+m\right)\right|}{s_{*}+m} m \mathrm{~d} m
\end{aligned}
$$

is positive definite on $\mathcal{E}$, and is positive definite and proper on $\mathcal{X}=\left(-s_{*}, \infty\right) \times\left(-x_{1 *}, \infty\right) \times\left(-x_{2 *}, \infty\right)$. Also,

$$
\dot{V} \leq-\aleph \frac{\tilde{s}^{2}}{s}+M\left(\tilde{s}, \tilde{x}_{1}, \tilde{x}_{2}\right) v
$$

along all trajectories of (23) in $\mathcal{E}$.

For the proof of Lemma 4, see Appendix A.2. The function $V$ is nonstrict because when $v=0$, the right side of its decay estimate is negative semidefinite as a function of $\left(\tilde{s}, \tilde{x}_{1}, \tilde{x}_{2}\right)$ but not negative definite.

\section{Completing the Proof of Theorem 1}

\subsection{Transforming $V$ into $V^{\sharp}$}

We first transform $V$ into an appropriate function $V^{\sharp}$ that is a strict Lyapunov functions when the delays are set to zero; this is a key step in our Lyapunov-Krasovskii construction. We refer to the trajectories of (23) with

$$
v=-\operatorname{sign}(\mho) \varepsilon_{1} \sigma\left(\varepsilon_{2} \tilde{Y}(t-\tau)\right)
$$

and with initial functions in $C_{\text {in }}$ as the closed loop trajectories of (23). Since $\mathcal{E}$ is forward invariant and globally attractive for this closed loop system, there is no loss in generality in assuming that the solution satisfies $\left(\tilde{s}(t), \tilde{x}_{1}(t), \tilde{x}_{2}(t)\right) \in \mathcal{E}$ for all $t \geq-2 \tau_{M}$. Since $a \in(0,1)$,

$$
\begin{aligned}
\tilde{x}_{1} & =\frac{-a \tilde{z}+a \tilde{s}+\tilde{Y}}{1-a}, \quad \tilde{x}_{2}=\frac{\tilde{z}-\tilde{s}-\tilde{Y}}{1-a}, \quad \text { and } \\
\frac{\tilde{s}}{s} & =\frac{-\tilde{s}^{2}}{s s_{*}}+\frac{\tilde{s}}{s_{*}} .
\end{aligned}
$$

Plugging (29) into (25) and collecting coefficients gives

$$
\begin{aligned}
M\left(\tilde{s}, \tilde{x}_{1}, \tilde{x}_{2}\right)= & L_{1}\left(s_{\text {in }}-s_{*}\right)\left[\frac{-\tilde{s}^{2}}{s s_{*}}+\frac{\tilde{s}}{s_{*}}\right]-\frac{L_{1}}{s} \tilde{s}^{2} \\
& +\left\{C_{3}-g_{1} s_{*}\left(s_{\text {in }}-s_{*}\right)\right\} \tilde{s} \\
& +\mho \tilde{Y}(t)-C_{3} \tilde{z}+g_{1} s_{*} \tilde{s}^{2} \\
= & -\frac{L_{1} s_{\text {in }}}{s_{*}} \frac{\tilde{s}^{2}}{s}+g_{1} s_{*} \tilde{s}^{2}+\bar{c}_{1} \tilde{s}+\mho \tilde{Y}(t)-C_{3} \tilde{z},
\end{aligned}
$$

where $\mho$ is defined in $(7)$ and

$$
C_{3}=\frac{1}{1-a} \frac{s_{*}}{D_{*}}\left[-K_{1} a+\frac{L_{1}-g_{1} s_{*}^{2}}{L_{2}-g_{2} s_{*}^{2}} K_{2}\right] .
$$


Substituting into (27) and recalling that $s \leq s_{\text {in }}$ on $\mathcal{E}$,

$$
\begin{aligned}
\dot{V} \leq & -\aleph \frac{\tilde{s}^{2}}{s}+\left[-\frac{L_{1} s_{\text {in }}}{s_{*}}+g_{1} s_{*} s\right] \frac{\tilde{s}^{2}}{s} v+\bar{c}_{1} \tilde{s} v \\
& +\mho \tilde{Y}(t) v-C_{3} \tilde{z} v \\
\leq & -\frac{9}{10} \aleph \frac{\tilde{s}^{2}}{s}+\bar{c}_{1} \tilde{s} v+\mho \tilde{Y}(t) v-C_{3} \tilde{z} v
\end{aligned}
$$

where the last inequality is from the second argument in the min defining our upper bound $\bar{\varepsilon}_{1}$ from (13). Here and in the rest of the proof, dots indicate time derivatives along the closed loop trajectories of (23), and we omit the arguments of our functions when they are clear.

Since we are assuming that $\left(\tilde{s}(t), \tilde{x}_{1}(t), \tilde{x}_{2}(t)\right) \in \mathcal{E}$ for all $t \geq-\tau_{M}$, we have

$$
|\tilde{Y}(t-\tau)|<1.1 s_{\text {in }} \quad \forall t \geq 0 .
$$

Therefore, our upper bound $\bar{\varepsilon}_{2}$ for $\varepsilon_{2}$ from (13) gives

$$
v=-\operatorname{sign}(\mho) \varepsilon_{3} \tilde{Y}(t-\tau) \quad \forall t \geq 0,
$$

where $\varepsilon_{3}=\varepsilon_{1} \varepsilon_{2}$. Substituting into (31) and then using

$$
\tilde{Y}(t-\tau)=\tilde{Y}(t)-\mathcal{I}_{t}(\tilde{Y}),
$$

where $\mathcal{I}_{t}(\tilde{Y})=\int_{t-\tau}^{t} \dot{\tilde{Y}}(m) \mathrm{d} m$, shows that for all $t \geq 0$,

$$
\begin{aligned}
\dot{V} \leq & -\frac{9}{10} \aleph \frac{\tilde{s}^{2}}{s}-|\mho| \varepsilon_{3} \tilde{Y}(t)^{2}-\operatorname{sign}(\mho) \bar{c}_{1} \varepsilon_{3} \tilde{s} \tilde{Y}(t) \\
& +\operatorname{sign}(\mho) C_{3} \varepsilon_{3} \tilde{z} \tilde{Y}(t)-C_{3} \operatorname{sign}(\mho) \varepsilon_{3} \tilde{z} \mathcal{I}_{t}(\tilde{Y}) \\
& +\left[\bar{c}_{1} \operatorname{sign}(\mho) \varepsilon_{3} \tilde{s}+|\mho| \varepsilon_{3} \tilde{Y}(t)\right] \mathcal{I}_{t}(\tilde{Y}) .
\end{aligned}
$$

Applying the triangle inequality $a b \leq a^{2} / 4+b^{2}$ to the last four terms in (34) gives

$$
\begin{aligned}
& -\operatorname{sign}(\mho) \bar{c}_{1} \varepsilon_{3} \tilde{s} \tilde{Y} \leq \frac{1}{4} \varepsilon_{3}|\mho| \tilde{Y}^{2}+\varepsilon_{3} \frac{\bar{c}_{1}^{2}}{|\zeta|{ }_{s}} \tilde{s}^{2} \text { and } \\
& \operatorname{sign}(\mho) C_{3} \varepsilon_{3} \tilde{z} \tilde{Y} \leq \frac{1}{16} \varepsilon_{3}|\mho| \tilde{Y}^{2}+4 \varepsilon_{3} \frac{C_{3}^{2}}{|\mho|} \tilde{z}^{2}
\end{aligned}
$$

and

$$
\begin{aligned}
-C_{3} \operatorname{sign}(\mho) \varepsilon_{3} \tilde{z} \mathcal{I}_{t}(\tilde{Y}) & \leq \frac{1}{4} \varepsilon_{3}^{2} \mathcal{I}_{t}^{2}(\tilde{Y})+C_{3}^{2} \tilde{z}^{2}, \\
\bar{c}_{1} \operatorname{sign}(\mho) \varepsilon_{3} \tilde{s} \mathcal{I}_{t}(\tilde{Y}) & \leq \frac{\aleph}{10} \frac{\tilde{s}^{2}}{s}+\frac{5 \bar{c}_{1}^{2} \varepsilon_{3}^{2} s}{2 \aleph} \mathcal{I}_{t}^{2}(\tilde{Y}), \\
|\mho| \varepsilon_{3} \tilde{Y} \mathcal{I}_{t}(\tilde{Y}) & \leq \frac{1}{16} \varepsilon_{3}|\mho| \tilde{Y}^{2}+4 \varepsilon_{3}|\mho| \mathcal{I}_{t}^{2}(\tilde{Y}) .
\end{aligned}
$$

Summing the inequalities (35)-(36) and collecting like terms, and then substituting the result into (34), gives

$$
\begin{aligned}
& \dot{V} \leq\left[-\frac{4}{5} \frac{\aleph}{s}+\varepsilon_{3} \frac{\bar{c}_{1}^{2}}{|\mho|}\right] \tilde{s}^{2}-\frac{5}{8} \varepsilon_{3}|\mho| \tilde{Y}^{2} \\
& +C_{3}^{2}\left(\frac{4 \varepsilon_{3}}{|\mho|}+1\right) \tilde{z}^{2}+\left[\frac{5 \bar{c}_{1}^{2} \varepsilon_{3}^{s}}{2 \aleph}+4 \varepsilon_{3}|\mho|+\frac{1}{4} \varepsilon_{3}^{2}\right] \mathcal{I}_{t}^{2}(\tilde{Y}) .
\end{aligned}
$$

Therefore, since $s \leq s_{\text {in }}$ on $\mathcal{E}$, we get

$$
\begin{aligned}
\dot{V} \leq & {\left[-\frac{4}{5} \aleph+\varepsilon_{3} \frac{\bar{c}_{1}^{2} s_{\text {in }}}{\mid \mho \mho}\right] \frac{\tilde{s}^{2}}{s}-\frac{5}{8} \varepsilon_{3}|\mho| \tilde{Y}^{2} } \\
& +C_{3}^{2}\left(\frac{4 \varepsilon_{3}}{|\mho|}+1\right) \tilde{z}^{2}+C_{4} \varepsilon_{3} \mathcal{I}_{t}^{2}(\tilde{Y}) \\
\leq & -\frac{3}{5} \aleph \frac{\tilde{s}^{2}}{s}-\frac{5}{8} \varepsilon_{3}|\mho| \tilde{Y}^{2}+C_{5} \tilde{z}^{2}+C_{4} \varepsilon_{3} \mathcal{I}_{t}^{2}(\tilde{Y}),
\end{aligned}
$$

where

$$
C_{4}=\frac{5 \bar{c}_{1}^{2} \varepsilon_{3} s_{\text {in }}}{2 \aleph}+4|\mho|+\frac{1}{4} \varepsilon_{3}, \quad C_{5}=C_{3}^{2}\left(\frac{4 \varepsilon_{3}}{|\mho|}+1\right),
$$

and the last inequality in (38) is by the first argument in the min in our bound $\bar{\varepsilon}_{3}$ for $\varepsilon_{3}$ from (13). Hence, the function $V^{\sharp}$ defined by

$$
V^{\sharp}=V+\frac{C_{5}+1}{D_{*}(1-0.5 p)} Q(\tilde{z})
$$

is a strict Lyapunov function when the delays are set to zero, because it satisfies

$$
\dot{V}^{\sharp} \leq-\frac{3}{5} \aleph \frac{\tilde{s}^{2}}{s}-\frac{5}{8} \varepsilon_{3}|\mho| \tilde{Y}^{2}-\tilde{z}^{2}+C_{4} \varepsilon_{3} \mathcal{I}_{t}^{2}(\tilde{Y}),
$$

by (15).

\subsection{Transforming $V^{\sharp}$ into $U_{1}$}

The next key step is to transform $V^{\sharp}$ into a function $U_{1}$ that satisfies the Lyapunov-Krasovskii conditions when $t \geq \tau_{M}$. By $(23)$,

$$
\dot{\tilde{x}}_{i}=\left[\frac{D_{*}\left[L_{i}-g_{i} s_{*} s\right]}{s_{*}\left(L_{i}+s+g_{i} s^{2}\right)} \tilde{s}+\operatorname{sign}(\mho) \varepsilon_{3} \tilde{Y}(t-\tau)\right] x_{i}
$$

for $i=1,2$ for all $t \geq 0$. The preceding observations give

$$
\begin{aligned}
& \left|\mathcal{I}_{t}(\tilde{Y})\right| \leq \varepsilon_{3} \int_{t-\tau}^{t}|\tilde{Y}(r-\tau)| Y(r) \mathrm{d} r \\
& +\int_{t-\tau}^{t}\left[\sum_{i=1}^{2} \frac{D_{*}\left[L_{i}-g_{i} s_{*} s(r)\right]}{s_{*}\left(L_{i}+s(r)+g_{i} s(r)^{2}\right)}|\tilde{s}(r)|\right] Y(r) \mathrm{d} r
\end{aligned}
$$

for all $t \geq \tau_{M}$.

We also have $x_{i}(t) \leq \frac{11}{10} s_{\text {in }}<\frac{3}{2} s_{\text {in }}$ for $i=1,2$. Hence, (42) and Assumption 2 imply that for all $t \geq \tau_{M}$,

$$
\begin{aligned}
& \mathcal{I}_{t}^{2}(\tilde{Y}) \leq 9(1+a)^{2} s_{\mathrm{in}}^{2}\left(\varepsilon_{3} \int_{t-\tau}^{t}|\tilde{Y}(r-\tau)| \mathrm{d} r\right)^{2} \\
& +9 s_{\mathrm{in}}^{2} \frac{D_{*}^{2}}{s_{*}^{2}}(1+a)^{2}\left(\int_{t-\tau}^{t}\left[\sum_{i=1}^{2} \frac{L_{i}-g_{i} s_{*} s(r)}{L_{i}+s(r)+g_{i} s(r)^{2}}|\tilde{s}(r)|\right] \mathrm{d} r\right)^{2} .
\end{aligned}
$$

Since $0<s \leq s_{\text {in }}$ on $\mathcal{E}$, Assumption 2 gives $0<L_{i}-$ $g_{i} s_{*} s(r) \leq L_{i}+s(r)+g_{i} s^{2}(r)$ on $\mathcal{E}$ for $i=1,2$, so we can replace $\left(L_{i}-g_{i} s_{*} s(r)\right) /\left(L_{i}+s(r)+g_{i} s(r)^{2}\right)$ by its upper bound 1 in the second integral. Hence,

$\mathcal{I}_{t}^{2}(\tilde{Y})$

$$
\begin{aligned}
& \leq C_{6}\left(\int_{t-\tau}^{t}|\tilde{s}(r)| \mathrm{d} r\right)^{2}+C_{7} \varepsilon_{3}^{2}\left(\int_{t-\tau}^{t}|\tilde{Y}(r-\tau)| \mathrm{d} r\right)^{2} \\
& \leq C_{6} s_{\mathrm{in}} \tau \int_{t-\tau}^{t} \frac{\tilde{s}(r)^{2}}{s(r)} \mathrm{d} r+C_{7} \varepsilon_{3}^{2} \tau \int_{t-\tau}^{t} \tilde{Y}(r-\tau)^{2} \mathrm{~d} r
\end{aligned}
$$

for all $t \geq \tau_{M}$, where

$$
C_{6}=36 s_{\mathrm{in}}^{2} \frac{D_{*}^{2}}{s_{*}^{2}}(1+a)^{2} \text { and } C_{7}=9(1+a)^{2} s_{\mathrm{in}}^{2}
$$

by Jensen's Inequality $\left(\int_{t-\tau}^{t} P(r) \mathrm{d} r\right)^{2} \leq \tau \int_{t-\tau}^{t} P^{2}(r) \mathrm{d} r$ for continuous functions $P$, and then multiplying by $s_{\text {in }} / s(r) \geq 1$ inside the first integral. Combining (43) with (40) gives

$$
\begin{aligned}
\dot{V}^{\sharp} \leq & -\frac{3}{5} \aleph \frac{\tilde{s}^{2}}{s}-\frac{5}{8} \varepsilon_{3}|\mho \mho| \tilde{Y}^{2}-\tilde{z}^{2} \\
& +\varepsilon_{3} C_{8} \tau_{M} \int_{t-\tau}^{t}\left\{\tilde{s}(r)^{2} / s(r)\right\} \mathrm{d} r \\
& +C_{8} \varepsilon_{3}^{3} \tau_{M} \int_{t-2 \tau}^{t} \tilde{Y}(r)^{2} \mathrm{~d} r
\end{aligned}
$$

for all $t \geq \tau_{M}$, where $C_{8}=C_{4}\left(C_{6} s_{\text {in }}+C_{7}\right)$. 
Then (40) implies that along the closed loop trajectories,

$$
\begin{aligned}
U_{1} \stackrel{\text { def }}{=} & V^{\sharp}\left(\tilde{s}, \tilde{x}_{1}, \tilde{x}_{2}\right) \\
& +2 \varepsilon_{3} C_{8} \tau_{M} \int_{t-2 \tau_{M}}^{t}\left(\int_{\ell}^{t}\left\{\tilde{s}(r)^{2} / s(r)\right\} \mathrm{d} r\right) \mathrm{d} \ell \\
& +2 C_{8} \varepsilon_{3}^{3} \tau_{M} \int_{t-2 \tau_{M}}^{t}\left(\int_{\ell}^{t} \tilde{Y}(r)^{2} \mathrm{~d} r\right) \mathrm{d} \ell
\end{aligned}
$$

satisfies

$$
\begin{aligned}
\dot{U}_{1} \leq & -\tilde{z}^{2}-\left[\frac{3}{5} \aleph-4 \varepsilon_{3} C_{8} \tau_{M}^{2}\right] \frac{\tilde{s}^{2}}{s} \\
& -\varepsilon_{3}\left[\frac{5}{8}|\mho|-4 C_{8} \varepsilon_{3}^{2} \tau_{M}^{2}\right] \tilde{Y}^{2}
\end{aligned}
$$

for all $t \geq \tau_{M}$, by (15). Our choice of $\bar{\varepsilon}_{3}$ in (13) gives $\bar{\varepsilon}_{3} \leq|\mho|$, and $\bar{c}_{2} \geq 1$, so simple calculations show that the constant $\bar{c}_{2}$ in (13) satisfies $C_{8} \leq \bar{c}_{2}$. It follows from our choice of the bound $\bar{\varepsilon}_{3}$ for $\varepsilon_{3}$ in (13) that

$$
\begin{aligned}
\dot{U}_{1} & \leq-\tilde{z}^{2}-\frac{\aleph}{5} \frac{\tilde{s}^{2}}{s}-\frac{\varepsilon_{3}|\mho|}{8} \tilde{Y}^{2} \\
& =-\left(\tilde{s}+\tilde{x}_{1}+\tilde{x}_{2}\right)^{2}-\frac{\aleph}{5} \frac{\tilde{s}^{2}}{s}-\frac{\varepsilon_{3}|\mho|}{8}\left(\tilde{x}_{1}+a \tilde{x}_{2}\right)^{2}
\end{aligned}
$$

for all $t \geq \tau_{M}$. Since $a \neq 1$, the right side of (48) is negative definite in $\left(\tilde{s}, \tilde{x}_{1}, \tilde{x}_{2}\right)$. The function $U_{1}$ is not a Lyapunov-Krasovskii functional in the usual sense because it only characterizes the behavior after time $\tau_{M}$, but it leads to the desired UGAS estimate, by standard Lyapunov-Krasovskii arguments; see Appendix A.3.

Remark 4 The same transformation we used in Section 5.1 works if $V$ is any function satisfying the conclusions of Lemma 4. Similarly, the transformation from Section 5.2 leads to the appropriate Lyapunov-Krasovskii decay estimate (48) for any proper and positive definite function $V^{\sharp}$ satisfying the estimate (40). The preceding stabilization problem cannot be solved using [17]. The paper [17] analyzed a class of systems $\dot{x}=f(t, x)+$ $g(t, x) u_{s}(t, x)$ that were known to be UGAS to 0 under a known feedback $u_{s}$, and then used a LyapunovKrasovskii argument to compute a constant $\bar{\tau}>0$ such that $\dot{x}(t)=f(t, x(t))+g(t, x(t)) u_{s}(t, x(t-\tau))$ is UGAS to 0 for all constant delays $\tau \in[0, \bar{\tau}]$. This result from [17] does not apply in our case because (a) we wish to construct output feedbacks that stabilize under arbitrarily large delay bounds and (b) the error dynamics (23) we are stabilizing has a drift term. See Remarks 4 and A.4 in [17]. While [12] and our other chemostat-related works in the references below also use strictification approaches to build strict Lyapunov functions, they do not apply in the situation we consider here, because the systems they cover have different forms, and because our earlier chemostat Lyapunov constructions are only for undelayed systems with Monod uptake functions.

Remark 5 Our only requirements on $\bar{c}_{2}$ are that it is at least $C_{8}=C_{4}\left(C_{6} s_{\text {in }}+C_{7}\right)$ and at least 1 , so there are many possible choices of $\bar{c}_{2}$. Another choice is as follows. Assume that $\bar{\varepsilon}_{3}<2 \min \left\{1, \aleph /\left(10 \bar{c}_{1}^{2} s_{\text {in }}\right)\right\}$, and that $s_{\text {in }} \geq 1$. Then, $C_{4} \leq 4|\mho|+1$, so we can take

$$
\bar{c}_{2}=(4|\mho|+1)\left\{36 s_{\text {in }}^{3} \frac{D_{*}^{2}}{s_{*}^{2}}(1+a)^{2}+9(1+a)^{2} s_{\text {in }}^{2}\right\} .
$$

We use this alternative formula for $\bar{c}_{2}$ in the next section.

\section{Example}

Consider the system (1) with the Haldane growth functions

$$
\mu_{1}(s)=\frac{6 s}{8+s+0.12 s^{2}} \text { and } \mu_{2}(s)=\frac{2 s}{1+s+0.04 s^{2}} .
$$

In our earlier notation, $K_{1}=6, L_{1}=8, g_{1}=0.12$, $K_{2}=2, L_{2}=1$, and $g_{2}=0.04$. See Fig. 1. Assumption 1 holds with $s_{*}=2.5, s_{\text {in }}=2.75$, and $D_{*}=1.33$. To check Assumption 2, notice that $L_{1}-g_{1} s_{*} s_{\text {in }}=7.18 \stackrel{\text { def }}{=}$ $\delta_{1}>0$ and $L_{2}-g_{2} s_{*} s_{\text {in }}=0.73 \stackrel{\text { def }}{=} \delta_{2}>0$. Also, $L_{2} g_{1}-$ $L_{1} g_{2}=\frac{3}{25}-\frac{8}{25}<0$. We must now choose the $x_{i *}$ 's so that Assumption 2 is satisfied and so that $s_{*}+x_{1 *}+$ $x_{2 *}=s_{\text {in }}=11 / 4$, which we do by selecting $x_{1 *}=0.19$ and $x_{2 *}=0.06$; in particular, this gives the necessary condition $\aleph>0$. Moreover,

$$
-K_{1}+\frac{L_{1}-g_{1} s_{*}^{2}}{L_{2}-g_{2} s_{*}^{2}} K_{2}=-6+\frac{8-\frac{3}{4}}{1-\frac{1}{4}} 2=\frac{40}{3}>0,
$$

so Assumption 2 holds for any constant $a \in(0,1)$. Also, $\mu_{1}\left(s_{\text {in }}\right)=1.42$ and $\mu_{2}\left(s_{\text {in }}\right)=1.36$ are strictly larger than $D_{*}=1.33$ so Assumption 3 is also satisfied. Hence, we can take $\left(s_{*}, x_{1 *}, x_{2 *}\right)=(2.5,0.19,0.06)$, and then Theorem 1 applies for any given delay bound $\tau_{M}>0$ and any constant $a \in(0,1)$.

We simulated (1) using the growth functions (49), $\left(s_{*}, x_{1 *}, x_{2 *}\right)=(2.5,0.19,0.06)$, and the feedback $(12)$ with $\mho$ from (7). Rounding our bounds $\bar{\varepsilon}_{i}$ to the second decimal places and using the alternative formula for $\bar{c}_{2}$ from Remark 5, we are able to choose $a=0.1, \varepsilon_{1}=0.01$, $\varepsilon_{2}=0.01$, and the delay $\tau=0.5$. We initialized the dynamics with $\left(s(t), x_{1}(t), x_{2}(t)\right) \equiv(2.5,1,0.1)$ on $[-1,0]$. See Figs. 2-4. Our simulation shows the rapid convergence of the state trajectories toward the equilibrium triple $\left(s_{*}, x_{1 *}, x_{2 *}\right)$, and so validates our stabilization theorem. In particular, our feedback design keeps the species levels from becoming very small, which would be dangerous in practice because small species levels can lead to extinction. Note too that the linearization of (1) around $\left(s_{*}, x_{1 *}, x_{2 *}\right)$ with the growth functions (49) and the constant dilution rate $D=D_{*}$ has a zero eigenvalue, and so is not even locally asymptotically stable with this constant controller.

\section{Conclusion}

The output feedback stabilization of chemostats with measurement delays in the dilution rate controller is a challenging problem that is of considerable interest. For one species chemostats, this problem was analyzed in [18]. Here we solved the problem for two species chemostats. Our stabilizing feedbacks depend only on a given linear combination of the species concentrations, which are measured with an unknown time delay. 


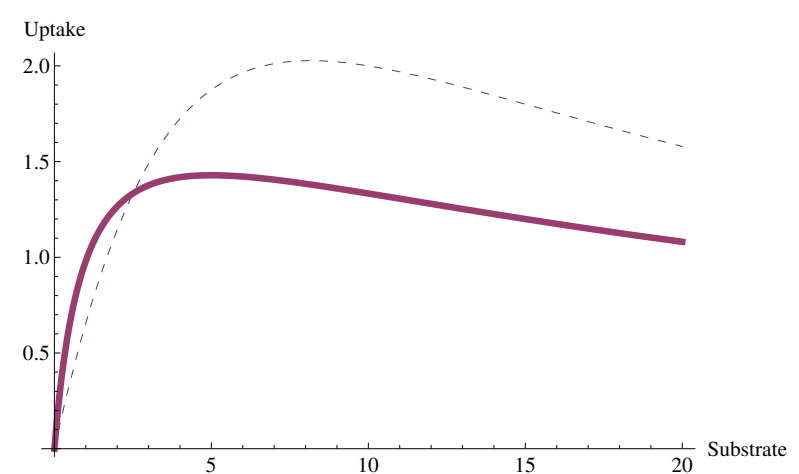

Fig. 1. $\mu_{1}$ (Dashed) and $\mu_{2}$ (Solid) from (49).

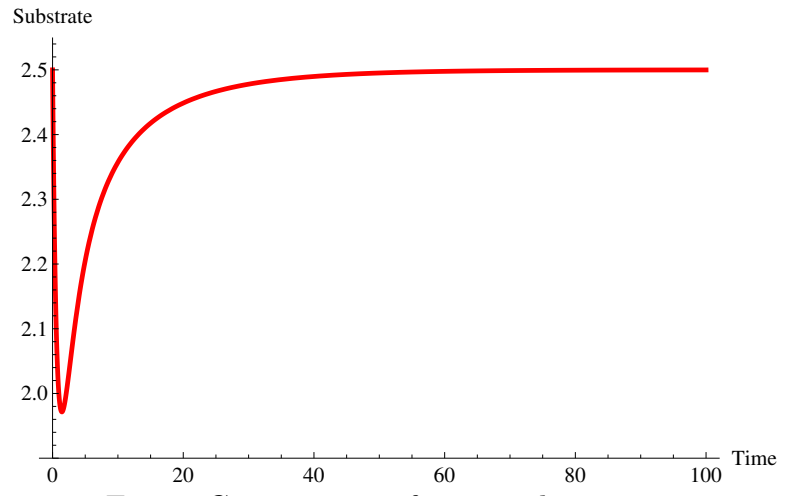

Fig. 2. Convergence of $s$ towards $s_{*}=2.5$.

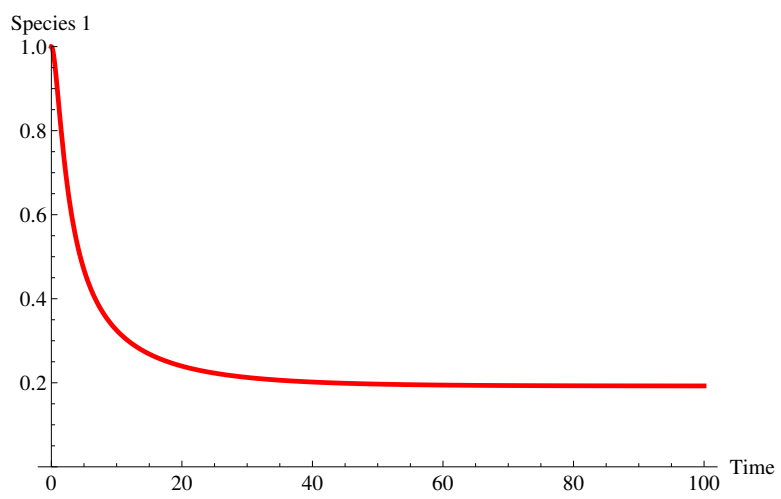

Fig. 3. Convergence of $x_{1}$ towards $x_{1 *}=0.19$.

One only knows an upper bound on the possible delays, and we allow the upper bound to be arbitrarily large. The stabilizing feedback depends on the known upper bound for the delay. When delays are present, the Poincaré-Bendixson and strict Lyapunov function approaches do not apply. Instead, we used a LyapunovKrasovskii approach. Our results cover cases where the growth functions are either Monod or Haldane. Our new Lyapunov-Krasovskii functional approach makes it possible to adapt the input-to-state stability arguments from [16] to show robustness to actuator errors. Due to space constraints, we omit this robustness analysis.

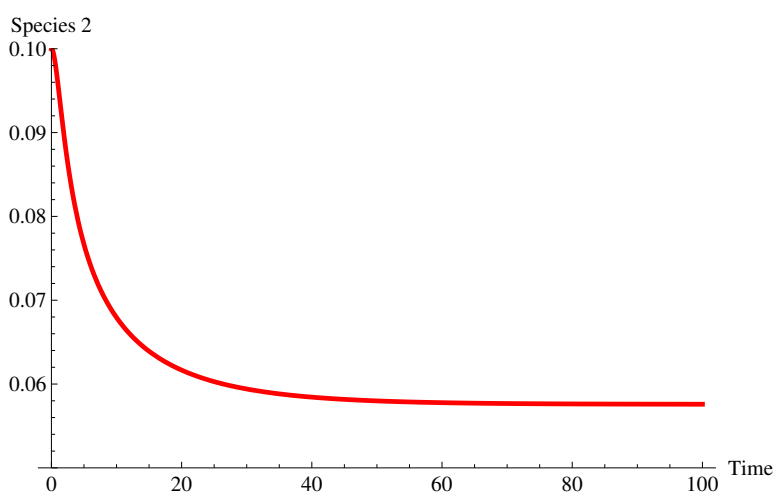

Fig. 4. Convergence of $x_{2}$ towards $x_{2 *}=0.06$.

\section{Appendices}

\section{A.1 Proof of Lemma 1}

Let $\left(s(t), x_{1}(t), x_{2}(t)\right)$ be any solution of (1) with the feedback (12), and with any initial function in $C_{\text {in }}$. One easily checks that each component of the solution stays positive. Also, the exponential stability of (15) provides a constant $T_{a}>0$ such that $\tilde{z}(t) \leq 0.1 s_{\text {in }}$, hence also $z(t) \leq \frac{11}{10} s_{\text {in }}$, for all $t \geq T_{a}$. We prove by contradiction that $s(t)$ enters $\left(0, s_{\text {in }}\right)$ in finite time. Suppose that $s(t) \geq$ $s_{\text {in }}$ for all $t \geq 0$. Then $\dot{s} \leq(1-0.5 p) D_{*}\left(s_{\text {in }}-s\right) \leq 0$ for all $t \geq 0$. This provides a constant $s_{L} \geq s_{\text {in }}$ such that $\lim _{t \rightarrow \infty} s(t)=s_{L}$. In fact, $s_{L}=s_{\text {in }}$, since otherwise $s$ would go to $-\infty$. We would then be able to find a time $T_{b} \geq T_{a}$ such that $\mu_{i}(s(t))>\mu_{i}\left(s_{\text {in }}\right)-0.25 p D_{*}$ for all $t \geq T_{b}$ and $i=1,2$, so (8) gives $\mu_{i}(s(t))-D \geq$ $\mu_{i}\left(s_{\text {in }}\right)-0.25 p D_{*}-(1+0.5 p) D_{*} \geq 0.25 p D_{*}$ for all $t \geq \bar{T}_{b}$ and $i=1,2$. This would imply that $x_{1}(t)$ and $x_{2}(t)$ go to $\infty$, contradicting the fact that $z(t)=s(t)+x_{1}(t)+x_{2}(t)$ is bounded. Hence, there exists a time $T_{c} \geq T_{a}$ such that $s\left(T_{c}\right) \in\left(0, s_{\text {in }}\right)$. This shows that $S$ is globally attractive for (1). Positive invariance follows because (I) $\dot{s}<0$ when $s=s_{\text {in }}$ and (II) $\left(-s_{\text {in }}, 0.1 s_{\text {in }}\right]$ is positively invariant for $(14)$, so $z(t)=\tilde{z}(t)+s_{\text {in }} \leq 1.1 s_{\text {in }}$ for all $t \geq 0$ if $z$ starts in $\left(0,1.1 s_{\text {in }}\right]$.

\section{A.2 Proof of Lemma 4}

Assumption 2 ensures that the function $V$ defined in (26) is positive definite on $\mathcal{E}$. To see why, notice that if $m \in\left(-s_{*}, s_{\text {in }}-s_{*}\right)$, then $L_{1}-g_{1} s_{*}\left(s_{*}+m\right) \geq L_{1}-$ $g_{1} s_{*} s_{\text {in }}=\delta_{1}>0$ and $m+s_{*}>0$, which give

$$
\int_{0}^{\tilde{s}} \frac{L_{1}-g_{1} s_{*}\left(s_{*}+m\right)}{s_{*}+m} m \mathrm{~d} m \geq \int_{0}^{\tilde{s}} \frac{\delta_{1}}{s_{*}+m} m \mathrm{~d} m .
$$

Moreover, $V$ is positive definite on $\mathcal{X}=\left(-s_{*}, \infty\right) \times$ $\left(-x_{1 *}, \infty\right) \times\left(-x_{2 *}, \infty\right)$, and $V\left(\tilde{s}, \tilde{x}_{1}, \tilde{x}_{2}\right) \rightarrow \infty$ as $\left(\tilde{s}, \tilde{x}_{1}, \tilde{x}_{2}\right) \in \mathcal{X}$ approaches any boundary point of $\mathcal{X}$ or as $\left|\left(\tilde{s}, \tilde{x}_{1}, \tilde{x}_{2}\right)\right| \rightarrow \infty$, which is the properness condition. However, $V\left(\tilde{s}, \tilde{x}_{1}, \tilde{x}_{2}\right)$ does not go to the infinity when $\tilde{s}$ approaches $s_{\text {in }}-s_{*}$. Nevertheless, this Lyapunov-like 
function, combined with the positive invariance of $\mathcal{E}$, will allow us to establish global asymptotic stability.

Since $\tilde{s}=s-s_{*}$, the expression (23) for the error dynamics and our choices (24) of $C_{1}$ and $C_{2}$ readily give

$$
\begin{aligned}
\frac{L_{1}-g_{1} s_{*} s}{s} \tilde{s} \dot{\tilde{s}}= & -\frac{L_{1}-g_{1} s_{*} s}{s} H(s) \tilde{s}^{2}-\frac{K_{1}\left(L_{1}-g_{1} s_{*} s\right)}{L_{1}+s+g_{1} s^{2}} \tilde{x}_{1} \tilde{s} \\
& +\frac{K_{2} g_{1} s_{*}}{L_{2}+s+g_{2} s^{2}} \tilde{x}_{2} \tilde{s}^{2}-\frac{K_{2}\left(L_{1}-g_{1} s_{*}^{2}\right)}{L_{2}+s+g_{2} s^{2}} \tilde{x}_{2} \tilde{s} \\
& +\tilde{s} \frac{L_{1}-g_{1} s_{*} s}{s}\left(s_{\text {in }}-s\right) v, \\
C_{1} \frac{\tilde{x}_{1} \dot{\tilde{x}}_{1}}{x_{1}}= & K_{1} \frac{L_{1}-g_{1} s_{*} s}{L_{1}+s+g_{1} s^{2}} \tilde{x}_{1} \tilde{s}-\frac{K_{1} s_{*}}{D_{*}} v \tilde{x}_{1}, \text { and } \\
C_{2} \frac{\tilde{x}_{2} \tilde{\tilde{x}}_{2}}{x_{2}}= & -\frac{\left(L_{1}-g_{1} s_{*}^{2}\right) K_{2} g_{2} s_{*}}{\left(L_{2}-g_{2} s_{*}^{2}\right)\left(L_{2}+s+g_{2} s^{2}\right)} \tilde{x}_{2} \tilde{s}^{2} \\
& +\frac{K_{2}\left(L_{1}-g_{1} s_{*}^{2}\right)}{L_{2}+s+g_{2} s^{2}} \tilde{x}_{2} \tilde{s}-\frac{L_{1}-g_{1} s_{*}^{2}}{L_{2}-g_{2} s_{*}^{2}} \frac{K_{2} s_{*}}{D_{*}} \tilde{x}_{2} v,
\end{aligned}
$$

where we used the relation $L_{i}-g_{i} s_{*} s=-g_{i} s_{*} \tilde{s}+\left(L_{i}-\right.$ $\left.g_{i} s_{*}^{2}\right)$ for $i=1$ to get the first equality and for $i=2$ to get the third equality. On $\mathcal{E}$, the sum of the preceding three equalities is $\dot{V}$ by definition. It follows from our choice (25) of the function $M$ and by canceling terms that on the positively invariant attractive set $\mathcal{E}$, we have

$$
\begin{aligned}
\dot{V}= & -\frac{L_{1}-g_{1} s_{*} s}{s} H(s) \tilde{s}^{2}+\frac{K_{2} g_{1} s_{*}}{L_{2}+s+g_{2} s^{2}} \tilde{x}_{2} \tilde{s}^{2} \\
& -\frac{\left(L_{1}-g_{1} s_{*}^{2}\right) K_{2} g_{2} s_{*}}{\left(L_{2}-g_{2} s_{*}^{2}\right)\left(L_{2}+s+g_{2} s^{2}\right)} \tilde{x}_{2} \tilde{s}^{2}+M\left(\tilde{s}, \tilde{x}_{1}, \tilde{x}_{2}\right) v \\
\leq & -\frac{\delta_{1}}{s} H(s) \tilde{s}^{2}+M\left(\tilde{s}, \tilde{x}_{1}, \tilde{x}_{2}\right) v \\
& +K_{2} s_{*}\left[\frac{L_{2} g_{1}-L_{1} g_{2}}{L_{2}-g_{2} s_{*}^{2}}\right] \frac{\left(x_{2}-x_{2 *}\right) \tilde{s}^{2}}{L_{2}+s+g_{2} s^{2}},
\end{aligned}
$$

where the inequality in (A.1) followed from Assumption 2 and the positivity of the lower bound (22).

By Assumption 2, $L_{2} g_{1}-L_{1} g_{2} \leq 0$ and $L_{2}-g_{2} s_{*}^{2} \geq$ $\delta_{2}>0$, since $s_{*} \in\left(0, s_{\text {in }}\right)$. We deduce that

$$
\begin{aligned}
& K_{2} s_{*}\left[\frac{L_{2} g_{1}-L_{1} g_{2}}{L_{2}-g_{2} s_{*}^{2}}\right] \frac{\left(x_{2}-x_{2 *}\right) \tilde{s}^{2}}{L_{2}+s+g_{2} s^{2}} \leq \\
& -K_{2} s_{*}\left[\frac{L_{2} g_{1}-L_{1} g_{2}}{\delta_{2}}\right] \frac{x_{2 *} \tilde{s}^{2}}{L_{2}+s+g_{2} s^{2}} .
\end{aligned}
$$

Also, $L_{2}+s+g_{2} s^{2} \geq s$ everywhere. Therefore, along all closed loop trajectories of (23) in $\mathcal{E}$, Assumption 2 and (22) give the nonstrict Lyapunov decay condition (27).

\section{A.3 UGAS Estimate}

Our decay estimate (48) makes it possible to construct the function $\beta \in \mathcal{K} \mathcal{L}$ in the uniform global asymptotic stability estimate for $(23)$ on $\mathcal{E}$, thereby quantifying the convergence of the trajectories to the equilibrium; we construct $\beta$ in this section 1 This can be done by a variant of the very last part of the proof of the main result

\footnotetext{
1 We say that a continuous function $\gamma:[0, \infty) \rightarrow[0, \infty)$ belongs to class $\mathcal{K}_{\infty}$ and write $\gamma \in \mathcal{K}_{\infty}$ provided it is strictly increasing and unbounded and $\gamma(0)=0$. We say that a continuous function $\beta:[0, \infty) \times[0, \infty) \rightarrow[0, \infty)$ is of class $\mathcal{K} \mathcal{L}$ provided (a) for each fixed $s \geq 0$, the function $\beta(\cdot, s)$ belongs to class $\mathcal{K}_{\infty}$, and (b) for each fixed $r \geq 0$, the function $\beta(r, \cdot)$ is non-increasing and $\beta(r, s) \rightarrow 0$ as $s \rightarrow \infty$.
}

in [17]. Here is a sketch. Our choice (46) of $U_{1}$ actually guarantees that along all of the closed loop trajectories of $(23)$ in $\mathcal{E}$, we have

$$
\begin{aligned}
\dot{U}_{1} \leq & -\tilde{z}^{2}-\frac{\aleph}{5} \frac{\tilde{s}^{2}}{s}-\frac{\varepsilon_{3}|\mho|}{8} \tilde{Y}^{2} \\
& -c_{o}\left(\int_{t-2 \tau_{M}}^{t} \frac{\tilde{s}(r)^{2}}{s(r)} \mathrm{d} r+\int_{t-2 \tau_{M}}^{t} \tilde{Y}^{2}(r) \mathrm{d} r\right)
\end{aligned}
$$

for all $t \geq \tau_{M}$, where $c_{o}=\varepsilon_{3} C_{8} \tau_{M} \min \left\{1, \varepsilon_{3}^{2}\right\}$. By reducing $c_{0}>0$ without relabeling, we can assume that

$$
\begin{aligned}
\dot{U}_{1} \leq-c_{0}\{ & \tilde{z}^{2}(t)+\tilde{Y}^{2}(t)+\frac{\tilde{s}^{2}(t)}{s(t)} \\
& +\int_{t-2 \tau_{M}}^{t}\left(\int_{\ell}^{t} \frac{\tilde{s}(r)^{2}}{s(r)} \mathrm{d} r\right) \mathrm{d} \ell \\
& \left.+\int_{t-2 \tau_{M}}^{t}\left(\int_{\ell}^{t} \tilde{Y}(r)^{2} \mathrm{~d} r\right) \mathrm{d} \ell\right\}
\end{aligned}
$$

along the closed loop trajectories of (23) in $\mathcal{E}$ for all $t \geq \tau_{M}$ (by bounding the double integrals in (A.4) with a constant times the single integrals in (A.3)). Let $L$ denote the quantity in braces in (A.4), and set $\mathcal{E}^{o}=\mathcal{E} \cap\left\{\left(-0.5 s_{*}, 0.5\left[s_{\text {in }}-s_{*}\right]\right) \times\right.$ $\left.\left(-0.5 x_{1 *}, 0.5 x_{1 *}\right) \times\left(-0.5 x_{2 *}, 0.5 x_{2 *}\right)\right\}$. Since $a \in(0,1)$, we can find a constant $c_{1}$ so that $U_{1} \leq c_{1} L$ whenever $\left(\tilde{s}(t), \tilde{x}_{1}(t), \tilde{x}_{2}(t)\right) \in \mathcal{E}^{o}$. Set $U_{2}=e^{U_{1}}-1$.

We can then find a constant $c_{2}>0$ so that $\dot{U}_{1}(t, \zeta) \leq$ $-c_{2} \min \left\{1, U_{1}(t, \zeta)\right\}$ and so also

$$
\dot{U}_{2}(t, \zeta) \leq-c_{2} U_{2}(t, \zeta)
$$

along all closed loop trajectories $\zeta$ of (23) for all $t \geq$ $2 \tau_{M}$; this follows by separately considering the cases $\left(\tilde{s}(t), \tilde{x}_{1}(t), \tilde{x}_{2}(t)\right) \in \mathcal{E}^{o}$ and $\left(\tilde{s}(t), \tilde{x}_{1}(t), \tilde{x}_{2}(t)\right) \in \mathcal{E} \backslash \mathcal{E}^{o}$, and then using the fact that $e^{u} u \geq e^{u}-1$ for all $u \geq 0$. We can find a function $\alpha_{0} \in \mathcal{K}_{\infty}$ and a modulus $\alpha_{1}$ with respect to $\mathcal{E}$ so that (i) $\alpha_{0}(|\zeta(t)|) \leq U_{2}(t, \zeta) \leq$ $\left|\alpha_{1}(\zeta(r))\right|_{\left[t-2 \tau_{M}, t\right]}$ for all $t \geq 0$ and (ii) $|\zeta(r)|_{\left[0,2 \tau_{M}\right]} \leq$ $\left|\alpha_{1}(\zeta(r))\right|_{\left[-2 \tau_{M}, 0\right]}$ for all closed loop trajectories $\zeta(t)=$ $\left(\tilde{s}(t), \tilde{x}_{1}(t), \tilde{x}_{2}(t)\right)$ of $(23)$ in $\mathcal{E} 2$ Therefore,

$$
|\zeta(t)| \leq \beta\left(\left|\alpha_{1}(\zeta(r))\right|_{\left[-2 \tau_{M}, 0\right]}, t\right)
$$

along all closed loop trajectories $\zeta$ of (23) in $\mathcal{E}$ for all $t \geq$ 0 , where $\beta \in \mathcal{K} \mathcal{L}$ is defined by $\beta(r, t)=\alpha_{0}^{-1}\left(\sup \left\{\alpha_{1}(q)\right.\right.$ : $\left.|q| \leq r, q \in \mathcal{E}\} \exp \left(c_{2}\left(2 \tau_{M}-t\right)\right)\right)+r \exp \left(c_{2}\left(2 \tau_{M}-t\right)\right)$.

\section{A.4 $\mho=0$ Case}

We show that if all of the assumptions of Theorem 1 hold except that $\mho=0$, and if $\mu_{1}$ and $\mu_{2}$ are different functions and $\tau=0$, then there does not exist a $C^{1}$ feedback $D$ that stabilizes the chemostat dynamics to the componentwise positive equilibrium $\left(s_{*}, x_{1 *}, x_{2 *}\right)$.

\footnotetext{
2 The existence of $\alpha_{1}$ follows from a slight variant of the proof of [17, Lemma A.1], applied on the invariant set $\mathcal{E}$. We use the sup norm notation $|\xi(r)| J \stackrel{\text { def }}{=} \sup \{|\xi(w)|: w \in J\}$ for the supremum of any continuous function on any interval $J$.
} 
To this end, first note that $\mho=0$ is equivalent to

$$
K_{1}\left(L_{2}-g_{2} s_{*}^{2}\right)=K_{2}\left(L_{1}-g_{1} s_{*}^{2}\right) .
$$

Since $\mu_{1}\left(s_{*}\right)=\mu_{2}\left(s_{*}\right)$ implies that

$$
\frac{1}{\left(L_{1}+s_{*}+g_{1} s_{*}^{2}\right)^{2}}=\frac{K_{2}^{2}}{K_{1}^{2}\left(L_{2}+s_{*}+g_{2} s_{*}^{2}\right)^{2}},
$$

we get

$$
\begin{aligned}
\mu_{1}^{\prime}\left(s_{*}\right) & =K_{1} \frac{L_{1}-g_{1} s_{*}^{2}}{\left(L_{1}+s_{*}+g_{1} s_{*}^{2}\right)^{2}}=\frac{K_{1}\left(L_{1}-g_{1} s_{*}^{2}\right) K_{2}^{2}}{K_{1}^{2}\left(L_{2}+s_{*}+g_{2} s_{*}^{2}\right)^{2}} \\
& =\frac{K_{2}\left(L_{1}-g_{1} s_{*}^{2}\right)}{K_{1}\left(L_{2}-g_{2} s_{*}^{2}\right)} \mu_{2}^{\prime}\left(s_{*}\right)=\mu_{2}^{\prime}\left(s_{*}\right),
\end{aligned}
$$

where the last equality used (A.6). On the other hand, simple calculations give

$$
\begin{aligned}
& K_{1} K_{2} \frac{s^{2}}{2} \mu_{i}^{\prime \prime}(s) \\
& =-K_{j} g_{i} \mu_{i}(s)^{2} s+\mu_{i}(s)\left[\mu_{i}^{\prime}(s)-\frac{\mu_{i}(s)}{s}\right] K_{j}\left(L_{i}-g_{i} s^{2}\right)
\end{aligned}
$$

for $j=1,2$ and $i=1,2$ with $i \neq j$. We deduce that

$$
\begin{aligned}
& K_{1} K_{2} \frac{s_{*}^{2}}{2} \mu_{1}^{\prime \prime}\left(s_{*}\right)= \\
& D_{*}\left[\mu_{1}^{\prime}\left(s_{*}\right)-\frac{D_{*}}{s_{*}}\right] K_{2}\left(L_{1}-g_{1} s_{*}^{2}\right)-K_{2} g_{1} D_{*}^{2} s_{*}
\end{aligned}
$$

and

$$
\begin{aligned}
& K_{1} K_{2} \frac{s_{*}^{2}}{2} \mu_{2}^{\prime \prime}\left(s_{*}\right)= \\
& D_{*}\left[\mu_{2}^{\prime}\left(s_{*}\right)-\frac{D_{*}}{s_{*}}\right] K_{1}\left(L_{2}-g_{2} s_{*}^{2}\right)-K_{1} g_{2} D_{*}^{2} s_{*} .
\end{aligned}
$$

Since $\mu_{2}^{\prime}\left(s_{*}\right)=\mu_{1}^{\prime}\left(s_{*}\right),($ A.6) and (A.9) give

$$
\begin{aligned}
& K_{1} K_{2} \frac{s_{*}^{2}}{2} \mu_{2}^{\prime \prime}\left(s_{*}\right)= \\
& D_{*}\left[\mu_{1}^{\prime}\left(s_{*}\right)-\frac{D_{*}}{s_{*}}\right] K_{2}\left(L_{1}-g_{1} s_{*}^{2}\right)-K_{1} g_{2} D_{*}^{2} s_{*} .
\end{aligned}
$$

By subtracting (A.10) from (A.8), we obtain

$$
\begin{aligned}
& K_{1} K_{2} \frac{s_{*}^{2}}{2}\left[\mu_{1}^{\prime \prime}\left(s_{*}\right)-\mu_{2}^{\prime \prime}\left(s_{*}\right)\right] \\
& =\left[-K_{2} g_{1}+K_{1} g_{2}\right] s_{*} D_{*}^{2} .
\end{aligned}
$$

Therefore, $\mu_{1}^{\prime \prime}\left(s_{*}\right)-\mu_{2}^{\prime \prime}\left(s_{*}\right)=0$ if and only if $-K_{2} g_{1}+$ $K_{1} g_{2}=0$. Assume that $-K_{2} g_{1}+K_{1} g_{2}=0$. Then we deduce from (A.6) that $K_{1} L_{2}=K_{2} L_{1}$. Since $\mu_{1}\left(s_{*}\right)=$ $\mu_{2}\left(s_{*}\right)$, we have $K_{1}\left(L_{2}+s_{*}+g_{2} s_{*}^{2}\right)=K_{2}\left(L_{1}+s_{*}+\right.$ $\left.g_{1} s_{*}^{2}\right)$. Using $K_{2} g_{1}=K_{1} g_{2}$ and $K_{1} L_{2}=K_{2} L_{1}$, we now get $K_{1}=K_{2}$. It follows that $g_{1}=g_{2}$ and $L_{1}=L_{2}$. Hence $\mu_{1}=\mu_{2}$. We conclude that when $\mho=0$, we have $\mu_{1}^{\prime \prime}\left(s_{*}\right)-\mu_{2}^{\prime \prime}\left(s_{*}\right)=0$ if and only if $\mu_{1}=\mu_{2}$.

Thus, we have established that if $\mu_{1} \neq \mu_{2}$ and $\mho=0$, then $\mu_{1}^{\prime}\left(s_{*}\right)=\mu_{2}^{\prime}\left(s_{*}\right)$ and $\mu_{1}^{\prime \prime}\left(s_{*}\right) \neq \mu_{2}^{\prime \prime}\left(s_{*}\right)$. Assume that $\mho=0$ and $\mu_{1} \neq \mu_{2}$, and let $\xi_{i}=\ln \left(x_{i}\right)$ and $\chi=\frac{\xi_{1}-\xi_{2}}{\mu_{1}^{\prime \prime}\left(s_{*}\right)-\mu_{2}^{\prime \prime}\left(s_{*}\right)}$ for all $x_{1}>0$ and $x_{2}>0$. Then

$$
\begin{aligned}
\dot{\chi} & =\frac{\left[\mu_{1}(s)-\mu_{1}\left(s_{*}\right)\right]-\left[\mu_{2}(s)-\mu_{2}\left(s_{*}\right)\right]}{\mu_{1}^{\prime \prime}\left(s_{*}\right)-\mu_{2}^{\prime \prime}\left(s_{*}\right)} \\
& =\frac{\int_{s_{*}}^{s}\left[\int_{s_{*}}^{r} \mu_{1}^{\prime \prime}(m) \mathrm{d} m-\int_{s_{*}}^{r} \mu_{2}^{\prime \prime}(m) \mathrm{d} m\right] \mathrm{d} r}{\mu_{1}^{\prime \prime}\left(s_{*}\right)-\mu_{2}^{\prime \prime}\left(s_{*}\right)} .
\end{aligned}
$$

We deduce that there is a constant $\varepsilon>0$ such that

$$
\frac{\int_{s_{*}}^{s} \int_{s_{*}}^{r}\left(\mu_{1}^{\prime \prime}(m)-\mu_{2}^{\prime \prime}(m)\right) \mathrm{d} m \mathrm{~d} r}{\mu_{1}^{\prime \prime}\left(s_{*}\right)-\mu_{2}^{\prime \prime}\left(s_{*}\right)} \geq 0
$$

for all $s \in\left[s_{*}-\varepsilon, s_{*}+\varepsilon\right]$. The fact that the equilibrium point $\left(s_{*}, x_{1 *}, x_{2 *}\right)$ cannot be locally uniformly asymptotically stabilized by a $C^{1}$ control (which necessarily would have $D=\mu_{1}\left(s_{*}\right)$ at the equilibrium, because $x_{1 *}>0$ ) now follows from the following lemma, applied to the $\left(\chi, s, \xi_{1}\right)$ dynamics in closed loop with any $C^{1}$ feedback and the equilibrium $\left(\chi_{*}, s_{*}, \xi_{1 *}\right)=\left(\left\{\ln \left(x_{1 *}\right)-\right.\right.$ $\left.\left.\ln \left(x_{2 *}\right)\right\} /\left\{\mu_{1}^{\prime \prime}\left(s_{*}\right)-\mu_{2}^{\prime \prime}\left(s_{*}\right)\right\}, s_{*}, \ln \left(x_{1 *}\right)\right)$ :

Lemma A.1 Let $\mathcal{O} \subseteq \mathbb{R}^{2}$ be any open set and

$$
\left\{\begin{aligned}
\dot{X} & =f_{1}(t, X, Z) \\
\dot{Z} & =f_{2}(t, X, Z), \quad(X, Z) \in \mathbb{R} \times \mathcal{O}
\end{aligned}\right.
$$

be any $C^{1}$ system. Let $\left(X_{e}, Z_{e}\right) \in \mathbb{R} \times \mathcal{O}$ be an equilibrium point of (A.13) and assume that there is a constant $\delta>0$ such that $\inf _{t \geq 0} f_{1}(t, X, Z) \geq 0$ for all $(X, Z) \in \mathbb{R} \times \mathcal{O}$ satisfying $\left|\left(X-X_{e}, Z-Z_{e}\right)\right| \leq \delta$. Then $\left(X_{e}, Z_{e}\right)$ is not a locally asymptotically stable equilibrium point of (A.13).

Proof: We prove the result by contradiction. Assume that $\left(X_{e}, Z_{e}\right)$ is a locally asymptotically stable equilibrium point of (A.13). Then we could find two positive constants $\varepsilon_{0} \in(0, \delta)$ and $\varepsilon_{1} \in(0, \delta)$ such that if $\left|\left(X_{0}-X_{e}, Z_{0}-Z_{e}\right)\right| \leq \varepsilon_{0}$, then the solution $(X(t), Z(t))$ of $\left(\right.$ A.13) with $\left(X_{0}, Z_{0}\right)$ as initial state satisfies $\left|\left(X(t)-X_{e}, Z(t)-Z_{e}\right)\right| \leq \varepsilon_{1}$ for all $t \geq 0$ and $\lim _{t \rightarrow \infty}(X(t), Z(t))=\left(X_{e}, Z_{e}\right)$. We choose the particular initial state $\left(X_{0}, Z_{0}\right)=\left(\varepsilon_{0}+X_{e}, Z_{e}\right)$. Then $\left|\left(X_{0}-X_{e}, Z_{0}-Z_{e}\right)\right| \leq \varepsilon_{0}$, so $f_{1}(t, X(t), Z(t)) \geq 0$ for all $t \geq 0$. Hence, $X(t) \geq \varepsilon_{0}+X_{e}$ for all $t \geq 0$, so $X(t)$ does not converge to $X_{e}$. We deduce that $\left(X_{e}, Z_{e}\right)$ cannot be a locally asymptotically stable equilibrium point of (A.13).

\section{References}

[1] M. Ballyk and E. Barany. The role of resource types in the control of chemostats using feedback linearization. Ecological Modelling, 211(1-2):25-35, 2006.

[2] E. Barany and M. Ballyk. Stabilization of chemostats using feedback linearization and reduction of dimension. In Proceedings of the American Control Conference (St. Louis, MO, June 10-12, 2009), pp. 2313-2318.

[3] P. De Leenheer and H.L. Smith. Feedback control for chemostat models. J. Math. Biol., 46(1):48-70, 2003.

[4] J.-L. Gouzé and G. Robledo. Feedback control for competition models with different removal rates in the chemostat. INRIA rapport de Recherche, 5555, 2005.

[5] J.-L. Gouzé and G. Robledo. Feedback control for nonmonotone competition models in the chemostat. Nonlinear Analysis: Real World Applications, 6(4):671-690, 2005.

[6] J.-L. Gouzé and G. Robledo. Robust control for an uncertain chemostat model. International J. Robust Nonlinear Control, 16(3):133-155, 2006. 
[7] S.B. Hsu. Limiting behavior for competing species. SIAM J. Applied Mathematics, 34(4):760-763, 1978.

[8] I. Karafyllis and C. Kravaris. Robust global stabilizability by means of sampled-data control with positive sampling sate. International J. Control, 82(4):755-772, 2009.

[9] I. Karafyllis, C. Kravaris and N. Kalogerakis. Relaxed Lyapunov criteria for robust global stabilization of nonlinear systems. International J. Control, 82(11):2077-2094, 2009.

[10] I. Karafyllis, C. Kravaris, L. Syrou and G. Lyberatos. A vector Lyapunov function characterization of input-to-state stability with application to robust global stabilization of the chemostat. European Journal of Control, 14(1):47-61, 2008.

[11] L. Mailleret, O. Bernard, and J.-P. Steyer. Nonlinear adaptive control for bioreactors with unknown kinetics. Automatica, 40(8):1379-1385, 2004.

[12] M. Malisoff and F. Mazenc. Constructions of Strict Lyapunov Functions. Communications and Control Engineering Series. Springer-Verlag London Ltd., London, UK, 2009.

[13] F. Mazenc, P. De Leenheer, and M. Malisoff. Stabilizing a periodic solution in the chemostat: A case study in tracking. In Proceedings of the 45th IEEE Conference on Decision and Control (San Diego, CA, 13-15 December 2006), pp. 17941799.

[14] F. Mazenc, M. Malisoff, and P. De Leenheer. On the stability of periodic solutions in the perturbed chemostat. Mathematical Biosciences and Engineering, 4(2):319-338, 2007.

[15] F. Mazenc, M. Malisoff, and J. Harmand. Further results on stabilization of periodic trajectories for a chemostat with two species. IEEE Trans. Automat. Contr., 53:66-74, Special Issue on Systems Biology, 2008.

[16] F. Mazenc, M. Malisoff, and J. Harmand. Stabilization in a two-species chemostat with Monod growth functions. IEEE Trans. Automat. Contr., 54(4):855-861, 2009.

[17] F. Mazenc, M. Malisoff, and Z. Lin. Further results on input-to-state stability for nonlinear systems with delayed feedbacks. Automatica, 44(9):2415-2421, 2008.

[18] G. Robledo. Feedback stabilization for a chemostat with delayed output. Mathematical Biosciences and Engineering, 6(3):629-647, 2009.

[19] H.L. Smith and P. Waltman. The Theory of the Chemostat. Cambridge University Press, Cambridge, UK, 1995.

[20] G. Wolkowicz and Z. Lu. Global dynamics of a mathematical model of competition in the chemostat: general response functions and differential death rates. SIAM J. Applied Mathematics, 52(1):222-233, 1992. 